\title{
How accurate is self reported birth weight among the elderly?
}

\author{
M Kemp, D Gunnell, M Maynard, G Davey Smith, S Frankel
}

The effect of early life influences on adult health is a central topic in current epidemiological research. For instance, growth and development in utero and in infancy have been linked to cardiovascular disease. ${ }^{1}$ In empirical research in this field, birth weight is often used as an indicator of health and nutrition in utero.

There are two main sources of birthweight data: birth records and the memories of subjects or their families, or both. Where the subjects are elderly, however, early birth records with recorded birth weights are not easy to find and the recovery rate is generally low. ${ }^{2}$ Where there are no surviving birth records with birthweight data and where the subject's parents cannot be asked, the subject's own testimony is the only possible source of such data.

But can self reported birth weight be relied upon in the absence of data from clinical or administrative sources? Birth weights recalled by mothers have been found to correlate highly with those found in official records. ${ }^{3}$ The accuracy of self reported birth weights, however, is less certain-some studies have reported a poor degree of correspondence between birth weights recorded in official records and self reported birth weights. ${ }^{4}$ Other researchers have found that birth weights from these two sources correlate reasonably well. ${ }^{5}$ Most investigations of the validity of self reported birth weight have typically used subjects who were middle aged or younger. In the investigation reported here, we use data collected in a study of childhood diet and health and disease in later life to estimate the accuracy of the self reported birth weights for an older age group of 57 to 77 year old women and men. It is important to note that the results reported are only relevant to studies involving the use of birthweight recall in older age groups. The older age of the subjects means that the probability of them having access to their mother's recall or being able to remember reports from their mothers is lower than younger age groups. In addition, for historical reasons, the likelihood of finding surviving birth or maternity records for a particular person tends to fall with that person's age.

Department of Socia Medicine, University of Bristol, Canynge Hall, Whiteladies Road, Bristol BS8 2PR

Correspondence to: Dr Gunnell

(D.J.Gunnell@bristol.ac.uk)

Accepted for publication 13 March 2000

\section{Methods}

The study subjects are members of the Boyd Orr cohort. ${ }^{6}$ This cohort comprises 4999 children drawn from 1352 families who underwent a one week assessment of family diet and health in 1937-39. The number of subjects has increased slightly since earlier reports of the cohort as a result of further searches of archived research records. Using the National
Health Service Central Register we have traced $85 \%$ of the original study members. As part of a recent follow up study, all subjects from the original sample of families who were traced and who were alive and living in Britain in 1997-98 were sent questionnaires requesting details of their current health, lifestyle and dietary habits $(n=3182)$. In the same questionnaire, subjects were asked to report their birth weight in pounds and ounces.

Recorded birth weights were collected separately through searches of archived birth and postnatal records in Aberdeen, Dundee, Edinburgh and London. Birthweight records were found for $10.0 \%$ (500) cohort members living in seven of the 16 study centres. ${ }^{2}$ Birth weights of 55 other subjects whose families had taken part in other surveys conducted by Sir John Boyd Orr were also retrieved but are not used in this analysis. We have previously compared the characteristics of those for whom birth weights were found and the entire cohort - the two groups are broadly similar according to age, sex, and childhood socioeconomic position.

Both self reported birth weights and those collected from official records were recorded in pounds and ounces; these have been converted to kilograms for the purpose of this analysis. To investigate the validity of the self reported birth weights we calculated the mean difference between self report and recorded birth weight, the limits of agreement and the correlation between the two measures. It is possible that respondents may have been better at recalling the "pound" part of their weight, but less likely to recall or record the "ounce" part correctly. To investigate this we also estimated the extent of approximate, rather than one to one, correspondence between the two sets of data. To assess the accuracy of classifying subjects into categories of low, average and high birth weight we divided subjects into three groups$(<2.5 \mathrm{~kg}, 2.5-3.5 \mathrm{~kg}$ and $>3.5 \mathrm{~kg})$ using the self report data and calculated the agreement between recorded and self report measures using the $\kappa$ statistic. Linear regression was used to investigate the influence of sociodemographic factors on accuracy of recall with differences between self report and actual birth weight as the dependent variable.

\section{Results}

Altogether 1647 (52\%) of the 3182 subjects sent questionnaires returned them and $46 \%$ (752) of these reported their birth weight. Compared with non-responders, questionnaire respondents tended to come from more affluent social backgrounds but there were no 


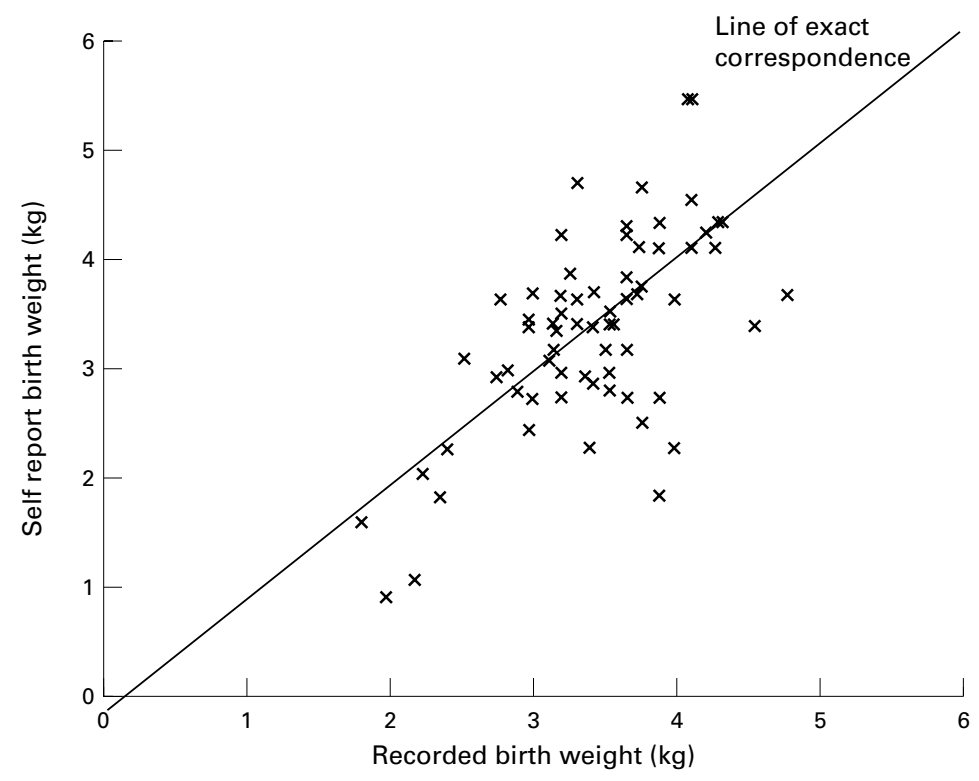

Figure 1 A graph showing the relation between self reported and recorded birth weight.

significant differences with respect to age, gender or childhood body mass index. ${ }^{7}$ Among the questionnaire respondents there were no significant differences in the sex, age or childhood economic position of those reporting their birth weights compared with those who did not.

Seventy three subjects, $1.5 \%$ of the original cohort, had both a recorded and a self reported birth weight. Their mean age was 64 years (SD 3.2 ) and $57 \%$ were women. The mean recorded birth weight was $3.42 \mathrm{~kg}$ (SD 0.58) and the mean self reported birth weight was $3.36 \mathrm{~kg}$ (SD 0.86) (mean difference $0.06 \mathrm{~kg}$ (95\% CI -0.09 to 0.22$)$; limits of agreement -1.23 to $1.35 \mathrm{~kg}$ ).

The correlation between self reported and actual birth weight was 0.64 (95\% CI 0.48 to $0.76)$. Eight subjects (11\%) reported their exact birth weight correctly and 30 (41\%) reported their weight in pounds correctly. When subjects were categorised into three birthweight groups $(<2.5 \mathrm{~kg}, 2.5-3.5 \mathrm{~kg}$, and $>3.5 \mathrm{~kg}$ ) using the self reported data, approximately two thirds of the subjects were placed in the correct category $(\kappa 0.43)$ indicating moderate agreement between the two sets of data using this method. Figure 1 shows that the association between self report and recorded birth weight is relatively weak and that low birthweight people tend to under-report their birth weight.

In univariable linear regression analyses, accuracy of recall was not significantly associated with age, sex and childhood socioeconomic position (measured as per capita household food expenditure in childhood). Subjects were also asked to identify where they had obtained the information about their birth weight: $56 \%$ had obtained this from their mother; $11 \%$ from a sister or brother; the remaining third of subjects either used some other source, did not state a source, but most said that they had guessed. Surprisingly, the accuracy of responses from those who obtained their birth weight from other family members was not significantly greater than those who obtained it from other sources. Because of the small sample size this analysis lacked power to detect potentially important effects.

\section{Discussion}

The low degree of association between self reported and actual birth weight indicates that the reliability of self reported birth weight in this group of elderly subjects is moderate to poor. It should also be noted in any interpretation of these results, that $54 \%$ of those who returned a completed questionnaire were unable to report their birth weight at all. The extent of misclassification and the low proportion of people of this age group who were able to state their birth weight suggests that the self reported birth weights of elderly people must be used with some caution in epidemiological research and that the applicability of such a method is limited.

In almost all cases, the source of subjects' reported birth weights was, either directly or indirectly, their mother. Recent research suggests that the maternal recall of birth weight is quite accurate. But where birth weight is self reported, there is an additional source of error: in the case where a subject's mother is no longer alive to ask, the validity of self reported birth weight crucially depends on the accuracy of the subject's (or sibling's) recall. An observed tendency to guess in combination with the accumulated errors in both the mother's recall and the subject's secondhand testimony would explain the results reported here.

We wish to acknowledge Walter Duncan, honorary archivist to the Rowett Research Institute, and Professor Philip James, director of the Rowett Research Institute for allowing us access to the original research records. We would also like to acknowledge Sara Bright and Sarah Pike for their assistance and the staff at the NHS Central Registers in Southport and Edinburgh. Funding: this work was funded by the World Cancer Research Fund.

Conflicts of interest: none.

1 Barker DJP The fetal and infant origins of adult disease. London: BMJ Publishing Group, 1992.

2 Kemp M, Gunnell D, Davey Smith G, et al. Finding and using inter-war maternity records. Social History of Medicine 1997;10:305-29.

3 Seidman DS, Slater PE, Ever-Hadani P, et al. Accuracy of mothers' recall of birth weight and gestational age. $B r \mathcal{F}$ Obstet Gynaecol 1987;94:731-5.

4 Little RE. Birth weight and gestational age: mothers' estimates compared with state and hospital records. Am $\mathcal{F}$ Public Health 1986;76:1350-1.

5 Sanderson M, Williams MA, White E, et al. Validity and reliability of subject and mother reporting of perinatal factors. Am J Epidemiol 1998;147:136-40.

6 Gunnell DJ, Frankel S, Nanchahal K, et al. Lifecourse exposure and later disease: a follow-up study based on a survey of family diet and health in pre-war Britain (1937-1939). Public Health 1996;110:85-94.

7 Gunnell DJ, Berney L, Holland P, et al. How accurately are height, weight and leg length reported by the elderly and how closely are they related to measurements recorded in childhood. Int 7 Epidemiol (in press). 